\section{Gluthathion S-transferase M1 and T1 polymorpisms in a group of Romanian glaucoma patients}

\section{DOI: $10.1515 / \mathrm{rrlm}-2015-0048$}

\section{To the Editor:}

Glaucoma is the second leading cause of blindness in the world, after cataract, according to the World Health Organization (WHO). According to some studies (1), the number of glaucoma patients worldwide is expected to increase up to 111.8 million by 2040 , with $74 \%$ of them suffering from primary open angle glaucoma (POAG), the most common type of glaucoma. Although, the exact number of glaucoma patients in Romania is not known, ophthalmologists describe an increasing prevalence of POAG among patients which often present in very late stages of the disease.

Although, high intraocular pressure (IOP) is recognized as being the main risk factor, the pathophysiology of POAG is considered multifactorial and multiple theories have been issued: mechanical damage due to high IOP, vascular dysregulation, altered intracranial pressure dynamics, glutamate excitotoxicity, nitric oxide dysregulation, astrocite reactivation, extracellular matrix remodelling, mutations in specific genes, changes in the mitochondrial genome, blood-retinal barrier breakdown and secondary low-grade inflammation, toxic effects and oxidative damage caused by reactive oxygen species (ROS) $(3,10)$.

Multiple in vivo and in vitro studies provide evidence for the involvement of reactive oxygen species (ROS) in POAG pathogenesis. An impairment of the eye's proficient antioxidant defence mechanisms, like reduced glutathione (GSH), seems to favour the progressive accumulation of oxidative damage which alters aqueous humour drainage pathways, increases IOP levels and triggers the 'pathogenic cascade' of POAG, ultimately leading to death of RGC through apoptosis. Glutathione-S-transferases (GSTs) are a family of phase II drug metabolising enzymes mostly known for their ability to catalyse the conjugation of reduced glutathione with a wide variety of electrophiles, including carcinogens and oxidative stress products. Null mutations of gluthathione S-transferase M1 (GSTM1) and T1 (GSTT1) are mainly responsible for the impairment or absence of GST enzymatic activity and have been involved in the pathogenesis of multiple disorders, including glaucoma (3).

GSTM1 and GSTT1 polymorphisms have been highly investigated as possible risk factors for POAG in various populations across the globe with inconsistent results (4-9). As none of these included a Romanian population, we thought it necessary to investigate the distribution of GSTM1 and GSTT1 polymorphisms in Romanian patients with POAG and in controls, to explore the possible association between GSTM1 and GSTT1 null genotypes and POAG in these patients. We then evaluated their impact on glaucoma subgroups according to family history, extra-ocular risk factors, surgical treatment and severity.

After recruiting 157 patients with POAG and 137 POAG glaucoma free patients or healthy controls who had given their informed consent, from the Ophthalmology Department of Cluj Emergency County Hospital, we conducted a cross-sectional, randomized case-control study. The tenets of the declaration of Helsinki were followed and the protocols for human experi- 
mentation and genetic testing were approved by the Ethical Commission of 'Iuliu Haţieganu' University of General Medicine and Pharmacy. All subjects recruited underwent a complete ophthalmological examination. POAG patients had to meet at least two of the following three criteria: (a) IOP above $21 \mathrm{mmHg}$; (b) pathological cupping of the optic disc; (C) a glaucoma hemifield test (GHT) outside normal limits with consistent visual field defects at the same location on at two consecutive visits and to have an open anterior chamber angle, at least grade III Schäfer.

Venous blood samples were obtained from all participants and DNA extraction was performed using Wizard Genomic DNA Purification Kit (Promega Madison, USA). A multiplex polymerase chain reaction protocol (adapted after Bid HK et al. (10)) was used for GSTM1 and GSTT1 genes genotyping which easily identifies the GSTT1 and GSTM1 homozygous null genotypes but, cannot distinguish between GSTM1 and GSTT1 homozygous and heterozygous positive genotypes.

For statistical analysis we used R software environment for statistics computing and graphics version 3.2.1 (rms functions package). Bivariate analysis were carried out using t test, Chi-square or Fisher exact tests. Ordinal, multinomial, and binary logistic regression analyses were also conducted. We used odds ratio (OR) and $95 \%$ associated confidence interval to highlight the size of tested associations. The level of significance in the multivariable logistic models and all two-sided tests was $\alpha=0.05$. Statistical significance was given by an estimated significance level, $\mathrm{p}<0.05$.

The two groups were comparable, as no significant differences were found with respect to age (student test for independent groups with unequal variances, $\mathrm{p}>0.05$ ) and sex (chi-square test, statistics $\chi 2=0.50, \mathrm{df}=1, \mathrm{p}=0.54$ ).
In this study we found no evidence of an association between GSTM1null or GSTT1null genotypes and POAG (GSTM1 p=0.335, GSTT1 $\mathrm{p}=0.483$ ). However, GSTM1null genotype showed a borderline association with the risk of POAG among women $(\mathrm{p}=0.055$, crude OR: 0.529 CI: 0.280-1.002), but not among men ( $p=0.414$, crude OR: 1.79 , CI: 0.596-5.38). Larger studies might provide statistically significant results and confirm a higher risk of developing POAG among women presenting GSTM1 null genotype. This could be due to genetic and hormonal differences. Similar but consistent results were found among women presenting lung cancer, where altered DNA repair mechanisms and hyperestrogenism are incriminated for the difference in disease susceptibility between women and men who smoke.

Our results are in agreement with the ones reported by Jansson et al who found no association between GSTM1 null genotypes and POAG in a Swedish population $(5,6)$. Similar results were also communicated for GSTM1 in a Brazilian, Mexican (4) and Iranian (5) populations and for GSTT1 in an Estonian, Turkish, Mexican, Brazilian and Iranian population $(5,6)$. Moreover, recent meta-analyses (7-9) found no significant association between GSTM1 null and GSTT1 genotypes and POAG in Caucasians. Only when analysis was stratified by ethnicity, a positive association was observed for GSTM1 null genotype in East Asians (9).

In contrast to our results, several studies have reported an increased risk of POAG in carriers of the GSTM1 null genotype in different populations (5-9). Only one study was able to document the association between GSTT1null genotype and glaucoma in a Turkish population. The previously mentioned meta-analyses reported a significantly increased risk of POAG in carriers of the double null genotype. We found no study to assess sex influence on GSTM1 and GSTT1 
null genotypes distribution among patients and controls. Many factors might account for the difference in results among similar studies, including different genetic, ethnic and environmental background and disparities among study design and methodology.

In order to asses a possible association between GST profiles and POAG, we examined the risk of glaucoma associated with combinations of genotypes. The reference group consisted of individuals with both low risk genotypes GSTM1 present/GSTT1 present. We found no statistically significant difference among the examined combinations even when stratified by sex.

We investigated a possible role of previous family history of glaucoma or personal history of extra-ocular conditions predisposing to POAG in determining genotype frequencies in the patient group, but found no evidence: personal history of extraocular conditions predisposing to POAG and GSTM1: Chi-square test, statistics $\chi 2=2.441 \mathrm{p}=0.137 \mathrm{OR}=0.37,95 \% \mathrm{CI}$ : 0.10-1.33; respectively GSTT1: Chi-square test, statistics $\chi 2=2.285 \mathrm{p}=0.152, \mathrm{OR}=1.9,95 \% \mathrm{CI}$ : 0.82-4.40; for family history of glaucoma and GSTM1 $\chi 2=0.123, \mathrm{p}=0.802, \mathrm{OR}=1.20, \mathrm{CI}=0.42$ 3.47 , GSTT1 $\chi 2=0.291, \mathrm{p}=0.674 \quad \mathrm{OR}=1.256$, 95\% CI: 0.54-2.88 (data not shown).

No statistically significant differences were found between patients who had or had not required surgical treatment for POAG with respect to GSTM1 null genotype: Chi-square test, $\mathrm{p}=0.202 \quad \mathrm{OR}=2.28,95 \% \mathrm{CI}: 0.77-6.72$, and GSTT1 null genotype: Chi-square test, $\chi 2=1.045$, $\mathrm{p}=0.441, \mathrm{OR}=0.58,95 \%$ CI: $0.21-1.63)$ (data not shown).

As a declining mean deviation (MD) value on the visual field is characteristic for POAG progression, its values can help classify POAG into stages according to MD severity scale in mild $(\mathrm{MD}<-6)$, moderate $(-12>\mathrm{MD} \geq-6)$ and ad- vanced $(\mathrm{MD} \geq-12)$ glaucoma. When evaluating factors influencing glaucoma severity according to MD staging scale, by ordinal logistic regression, we found that GSTM1 null genotype carriers had a higher risk of developing a moderate or advanced disease as compared to patients with the wild type genotype $(\mathrm{p}=0.002, \mathrm{OR}=3.362$, 95\% CI: 1.45-7.77) (data not shown)). Moreover, when we examined GSTM1-GSTT1 combinations, patient carriers of the double null genotype had a two-fold increased risk of moderate or severe glaucoma $(\mathrm{p}=0.003, \mathrm{OR}=2.14,95 \%$ CI: 0.70-6.49) (data not shown).

When multiple factors were evaluated as possible predictors of moderate and advanced glaucoma by multinomial logistic regression, moderate glaucoma was greatly influenced by GSTM1null genotype ( $\mathrm{p}=0.002$, OR:9.17, 95\% CI: 2.23-37.61), whereas advanced glaucoma was more frequent in patients dealing with glaucoma for more than 10 years $(\mathrm{p}=0.02, \mathrm{OR}: 2.37$, 95\% CI:1.17-4.78) and in GSTM1 null genotype carriers although we found only a trend to statistical significance $(\mathrm{p}=0.10, \mathrm{OR}=2.48, \mathrm{CI}=0.84$ 7.3)(Table I). This might be due to the small sample size in glaucoma severity subgroups. Larger studies might confirm the association and reach valuable statistical parameters. We found no other study to investigate the impact of GSTM1 and GSTT1 null polymorphisms on disease severity.

In conclusion, our study found no statistically significant association between GSTM1 or GSTT1null genotypes and POAG possibly due to the limited sample size. Our results suggest that the impairment of GSTM1 enzymatic activity may influence POAG progression. Restoring their proper function might slower the degenerating path of POAG and provide a potential therapeutic target for POAG. Further studies of greater amplitude need to be conducted in multiple populations in order to elucidate glaucoma pathogenesis and progression. 
Table I. The results of univariate and multivariate logistic regression for types of glaucoma Moderate vs. Early glaucoma

\begin{tabular}{lccc}
\hline Predictors & $\mathrm{p}^{* *}$ & Crude OR & $95 \%$ CI \\
\hline GSTM1(null/present) & 0.003 & 7.95 & $2.06-30.74$ \\
\hline GSTT1(null/present) & 0.324 & 0.52 & $0.14-1.92$ \\
\hline Family history (present/absent) & 0.891 & 1.12 & $0.22-5.62$ \\
\hline Extraocular risk factors (present/absent) & 0.080 & 0.30 & $0.08-1.16$ \\
\hline Duration of disease & 0.583 & 0.55 & $0.06-4.75$ \\
\hline The multivariable logistic model & $\mathbf{p}^{* *}$ & Adjusted OR & $\mathbf{9 5 \%}$ CI \\
\hline GSTM1(null/positive) & $\mathbf{0 . 0 0 2}$ & $\mathbf{9 . 1 7}$ & $\mathbf{2 . 2 3 - 3 7 . 6 1}$ \\
\hline GSTT1(null/positive) & 0.362 & 0.52 & $0.13-2.12$ \\
\hline Disease duration $(\geq 10$ years/<10) & 0.548 & 0.49 & $0.05-4.91$ \\
\hline APP (present/absent) & 0.078 & 0.26 & $0.06-1.16$ \\
\hline Intercept of model & 0.021 & - & - \\
\hline
\end{tabular}

Advanced vs. Early glaucoma

\begin{tabular}{lccc}
\hline Predictors & $\mathbf{p}^{* *}$ & Crude OR & $\mathbf{9 5 \%}$ CI \\
\hline GSTM1(null/present) & 0.063 & 2.65 & $0.95-7.39$ \\
\hline GSTT1(null/present) & 0.126 & 0.48 & $0.18-1.23$ \\
\hline Family history (present/absent) & 0.931 & 1.05 & $0.32-3.43$ \\
\hline Extraocular risk factors (present/absent) & 0.411 & 1.90 & $0.41-8.79$ \\
\hline Duration of disease & 0.011 & 2.42 & $1.23-4.77$ \\
\hline The multivariable logistic model & $\mathbf{p}^{* *}$ & $\mathbf{A d j u s t e d ~} \mathbf{O R}$ & $\mathbf{9 5 \%} \mathbf{C I}$ \\
\hline GSTM1(null/positive) & 0.10 & 2.48 & $0.84-7.3$ \\
\hline GSTT1(null/positive) & 0.110 & 0.44 & $0.16-1.20$ \\
\hline Extraocular risk factors $(\geq 10$ years/<10) & 0.02 & 2.37 & $1.17-4.78$ \\
\hline Extraocular risk factors (present/absent) & 0.553 & 1.61 & $0.33-7.73$ \\
\hline Model intercept & $\mathbf{0 . 0 0 2}$ & - & - \\
\hline *estimated unstandardized regression coefficients; SE=standard error;CI=confidence interval & & \\
** Wald's test adjusted p-value & & &
\end{tabular}

\author{
Anda Maria Ster ${ }^{*}$, Felicia Maria Petrisor ${ }^{1}$, \\ Cristina Stan', Victor Ioan Pop ${ }^{3}$ \\ 1. "Iuliu Hatieganu" University of General \\ Medicine and Pharmacy \\ 2. "Iuliu Hatieganu” University of General \\ Medicine and Pharmacy, Ophthalmology De- \\ partment
}

3. "Iuliu Hatieganu” University of General Medicine and Pharmacy, Clinical Genetics Department

* Corresponding author: Anda Maria Ster, email: ster.anda@yahoo.com

Conflict of interest: None 


\begin{abstract}
Abbreviations
POAG- primary open angle glaucoma

RGC- retinal ganglion cells

GST Glutathione S Transferase

GSTM1 - Glutathione S Transferase M1

GSTM1- Glutathione S transferare T 1

PCR- polymerase chain reaction
\end{abstract}

Received: $9^{\text {th }}$ July 2015; Accepted: $1^{\text {st }}$ December 2015; Published: 14 ${ }^{\text {th }}$ December 2015

\section{References}

1. Tham YC, Li X, Wong TY, Quigley HA, Aung T, Cheng CY. Global Prevalence of Glaucoma and Projections of Glaucoma Burden though 2040 A Systematic Review and Meta-Analyis. Ophthalmol. 2014;121:2081-90. DOI: 10.1016/j.ophtha.2014.05.013

2. Janssen SF, Gorgels TG, Ramdas WD, Klaver CC, van Dujin CM, Jansonius NM, et al. The vast complexity of primary open angle glaucoma: Disease genes, risks, molecular mechanisms and pathobiology. Prog Retin Eye Res. 2013 Nov;37:31-67. DOI: 10.1016/j.preteyeres.2013.09.001

3. Simionescu R, Cherecheanu AP, Voinea L, Sfrenț-Cornățeanu. TNF- $\alpha$ Gene Polymorphisms and Primary Open Angle Glaucoma in Romanian Population. Rev Romana Med Lab. 2015;23(1):47-58.

4. Buentello-Volante B, Elizondo-Olascoaga C, Miranda-Duarte A, Guadarrama-Vallejo D, Cabral-Macias J, Zenteno JC. Association study of multiple gene polymorphisms with the risk of adult-onset primary open-angle glaucoma in a Mexican population.
Exp Eye Res. 2013 Feb;107:59-4. DOI: 10.1016/j. exer.2012.11.013

5. Safa FK, Shahsavari G, Abyaneh RZ. Glutathione s-transferase M1 and T1 genetic polymorphisms in Iranian patients with glaucoma. Iran J Basic Med Sci. 2014 May;17(5):332-6.

6. Silva CTX, Costa NB, Silva KSF, Silve RE, Moura KKVO. Association between primary open angle glaucoma and genetic polymorphisms GSTM1/GSTT1 in patients from Goiâna Central-West region of Brazil. Genet Mol Res. 2014 Oct;13(4):8870-5. DOI: 10.4238/2014.October.31.2

7. Yu Y, Weng Yu, Guo J, Chen G, Yao K. Association of Glutathione S transferases Polymorphisms with Glaucoma: A Meta-Analysis. PloS ONE[serial online] 2013 [cited 2015 June]8(1):e54037. Available from URL: http://www.ncbi.nlm.nih.gov/pubmed/23342067.

8. Huang W, Wang W, Zhou M, Chen S, Zhang X. Association of glutathione S-transferase polymorphisms (GSTM1 and GSTT1) with primary open-angle glaucoma: An evidence based meta-analysis. Gene. 2013 Sept;526(2):80-6. DOI: 10.1016/j.gene.2013.05.032

9. Lu Y, Shi Y, Yin J, Huang Z. Are Glutathione S-transferase polymorphisms (GSTM1,GSTT1) associated with primary open angle glaucoma? A meta-analysis. Gene. 2013 Sept;527(1):311-5. DOI: 10.1016/j. gene.2013.06.031

10. Bid HK, Konwar R, Saxena M, Chaudhari P, Agrawal CG, Banerjee $\mathrm{M}$, et al. Association of glutathione S-transferase (GSTM1, T1 and P1) gene polymorphisms with type 2 diabetes mellitus in north Indian population. J Postgrad Med. 2010 Jul;56(3):176-1. DOI: $10.4103 / 0022-3859.68633$ 